\title{
美国智能雨洪管理途径与发展前景研究
}

\section{Current Instruments and Future Development of Intelligent Stormwater Management in the US}

摘要: 随着雨洪管理日益受到关注和智能技术的迅猛发展, 智能雨洪 管理成为美国各级政府和相关部门对雨洪管理实现有效和实时调控的 热点发展方向。通过在基础设施上安装智能传感组件, 与管理中心发 生交互, 完成雨洪信息搜集、分析、处理、控制和调整, 智能雨洪管 理扩展了基础设施的功能范围, 增强了自动化操作和管理的灵活性, 降低了长期维护成本和人力投入, 有利于提升雨洪管理的效率和成 果。本文以智能雨洪管理的发展背景为切入点, 辩析智能雨洪管理的 相关概念, 并结合实际案例分析了美国雨洪管理的应用现状、发展 重点、现实阻碍和有效推广途径, 以期对我国未来雨洪管理的智能 化提供借鉴。

Abstract: With a broad attention on stormwater management and the rapid development of intelligent technology, intelligent stormwater management provides opportunities to governments at all levels to integrate the real-time control in stormwater management. By installing sensors and controls on stormwater infrastructure with connection to a central management system, intelligent stormwater management fulfills an entire process of collection, analysis, control, operation and adjustment in the system. It improves infrastructure capability, enhances auto-operation and flexibility, saves longrun labor and maintenance cost, and increases the efficiency of stormwater management. Based on the demonstration of background and key concepts, this paper investigates the current status, implementing opportunities, potential barriers, and promotion channels of intelligent stormwater management in the US. It provides insightful experience and lessons on how to use intelligent stormwater management in China.

关键词: 智能感测; 智能控制; 雨洪基础设施

Keywords: Intelligent Sensoring; Intelligent Control; Stormwater Infrastructure

国家自然科学基金项目 (41501169)

\section{引言}

全球气候变化、快速城市化和环境保护要求提升给传统 雨洪管理模式带来极大挑战。在美国, 频发的极端天气和自 然灾害对城市发展造成严重威胁。此外, 快速城市化使得城 市传统排水系统压力增大, 而相关法律法规又逐年提高雨洪 管理的环保要求和生态维护标准 ${ }^{[1.3]}$ 。这一系列外部因素驱 使雨洪管理系统由静态到动态、从被动到主动、从零散到系 统的转变升级。随着感测、控制、通信、数据处理等智能技 术的迅速发展及其应用成本的显著降低, 智能雨洪管理成为 美国各级政府和相关部门对雨洪管理实现有效实时调控的热 点发展方向。对比传统雨洪管理, 智能雨洪管理的实时监控、 有预见性的控制、危机应对和反应, 以及自我维护等一系列 技术应用可以提高雨洪管理的效率和使用弹性 ${ }^{[4]}$ 。

尽管相关的智能技术已经相对成熟, 但是美国当前的智 能雨洪管理尚处于探索阶段, 应用多集中在政策和监管的需 求分析和学术研究, 实地大规模的采用和推广比较有限。已 有的实践中, 智能技术被运用到综合排水系统中, 监控、控 制和减少不必要的未处理的雨污合流排放频率 ${ }^{[5]}$ 。亦有机构 将集成无线感测器用于水体质量的监控、控制和管理 ${ }^{[6]}$ 。

本文首先分析智能雨洪管理在美国的发展背景, 辨析智 能雨洪管理的相关概念 ; 然后结合实际案例阐述美国雨洪管 理的实施途径、发展空间、现实阻碍和有效推广途径; 最后 结合我国当前城市雨洪管理问题, 提出美国城市智能雨洪管 理经验对我国未来雨洪管理的借鉴意义。

\section{1 智能雨洪管理的发展背景}

智能技术的迅猛发展和成熟, 以及相关技术在水利系统 中的有益尝试, 为雨洪管理系统的升级提供了新的思路和有 力的技术保障。归纳来讲, 智能雨洪管理的发展背景源于两 
股力量的互相作用: 一是对雨洪管理系统的新需求, 二是日 益成熟的智能技术和其在水利系统中的应用。

\section{1 对雨洪管理系统的新需求}

面对全球气候变化、快速城市化、环境保护的多重压力, 雨洪管理系统需要在原有的控制和利用雨洪的基础上完成由 静态到动态、由被动到主动、由零散到系统的一系列转变, 从而积极应对极端天气和自然灾害, 高效合理地处理快速城 市化带来的过量径流, 降低水环境污染和维护水域生态系统。

第一, 由静态到动态的转变。由于自身静态处理能力的 限制, 使得当前的雨洪管理系统对多变的气候状况和演进的 土地利用形式缺乏动态的适应 ${ }^{[7]}$ 。一方面, 雨洪量的处理和 管理能力相对固定, 不能及时快速地应对多变和突发的极端 气候和灾害事件。另一方面, 缺乏与周围环境的动态交互, 无 法实时和准确监控相关的环境参数。因此, 把雨洪量的不确定 性和同周边环境的协调性纳人动态管理是未来发展的需要。

第二, 由被动到主动的转变。传统的雨洪管理需要提高 自身的韧性来应对极端天气和自然灾害 ${ }^{[8]}$ 。系统可以通过及 时发出预警信号, 作出合理、有预见性的决策, 主动控制和 管理雨洪在时间和空间分布的不均衡。比如, 结合天气预报 和系统自身情况, 提前清空 (或蓄满) 部分水库来应对可能 的洪水 (或干旱)。主动的应对和管理可以在现有基础设施 不扩建的基础上, 极大地提高其功能性。

第三, 由零散到系统的转变。现有零散的相对孤立的 各个雨洪基础设施, 不能有效地应对频发的、极端的自然灾 害, 完成水域系统内的环境保护合作。为了充分发挥现有水 利设施的作用, 更合理地管理雨洪, 需要把现有的本地区内、 临近地区间和归属同一水域的各个雨洪管理设施和子系统有 机地联通起来, 形成交互的大网络。

\section{2 智能技术的发展和应用}

近年来, 智能技术迎来了快速和卓越的发展。传感器的 更新升级、无线通信设备的日益成熟、大型数据平台的搭建 和管理, 以及各个智能组件成本的显著降低, 都为智能技术 的推广提供了技术保障和经济可行性 ${ }^{[7]}$ 。智能技术和雨洪管 理系统的有机结合为雨洪系统的升级提供了新思路（图 1), 集中体现在以下几个方面。

首先, 实时监测。智能感测和监测组件可以广泛用于监 测雨洪管理宏观和微观的参数, 有利于实时掌握基础设施的 运行情况以及设施和周围环境的交互。宏观上, 包括系统各 个环节的操作和运行是否顺畅, 以及各个节点的已用和可用 空间; 微观上, 包括径流量、降水量、土壤湿度和成分, 当 地的温度和风速等等。另外, 要特别提出智能雨洪管理在环
境和生态保护方面的潜在贡献。智能组件可以用于监控水体 质量 ${ }^{[5,6]}$, 连续测量水体营养成分、金属含量和细菌等有关 水质管理的重要数据, 为水域生态环境治理提供宝贵的第一 手的资料。

其次, 自动操作和维护。在已有的水利基础设施上安装 低成本可靠的感测器和控制器可以提高对城市径流的自动管 理 ${ }^{[7]}$ 。智能控制组件包括水阀、水门和水泵等的应用, 可以 有效提高系统的自动控制功能, 成本低、效率高、控制及时。 另外, 根据掌握的系统信息, 智能技术还可以在必要时准确 和高效地找出故障点, 完成自动维护。

再次, 预见性决策和系统性管理。随着数据存储和分析 功能的加强, 智能雨洪管理可以汇总系统实时的数据, 结合 天气环境预报作出预见性的决策和管理。比如, 提前控制 智能水闸清空水库, 有效增加系统的蓄水能力, 应对未来的 暴雨天气; 针对可能的持续高温天气, 预见性地调节系统的 功能模式 (从排水到蓄水的转换), 确保各个部分节水正常 运作。

综合雨洪管理的新需求和智能技术的特性, 智能雨洪管 理扩展了基础设施的功能范围, 增强了自动化操作和管理的 灵活性, 同时有效地降低了长期维护成本和人力投人, 有利 于提升雨洪管理的效率和成果。

\section{2 智能雨洪管理的相关概念}

\section{1 智能雨洪管理}

广义上讲, 智能雨洪管理指的是将智能技术和智能发展 思路运用到雨洪管理的各个环节, 比如基础设施的操作和维 护、组织机构和人力资源管理、预警和应对决策和控制、以 及各个子系统相互协调联系等。本文重点关注的是所谓狭义 的智能雨洪管理, 也是雨洪管理的核心, 即通过智能雨洪基

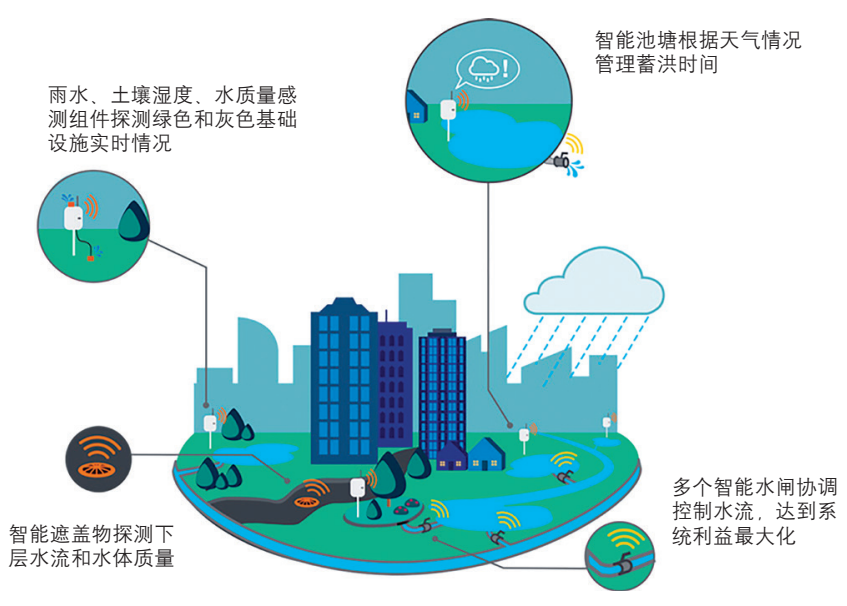

图 1 智能技术在雨洪管理系统的潜在应用 资料来源 : 参考文献 [7] 
础设施对水量和水质进行实时和有效的科学管理。智能雨洪 管理的过程可以归纳为：通过在雨洪基础设施上安装智能传 感和控制组件, 与管理中心发生交互, 完成对系统内部和外 部有关雨洪信息的搜集、分析、处理、控制和调整，对雨洪 基础设施进行实时自动操作、维护和科学管理（图 2)。

有关智能雨洪基础设施的概念要点归纳如下。首先, 与 雨洪管理相关的智能技术范围广泛, 涉及感测、控制、通信 和计算等诸多环节。所采用的智能技术和设备不局限于某一 类或者几类特定的类别, 而是根据管理系统的需要灵活选取。 其次, 与智能技术结合的雨洪基础设施内涵也很广泛。既包 括传统的灰色水利基础设施, 又包括近些年重点推广的具有 环保意义的绿色基础设施; 既可以是新设计和建造的基础设 施, 也可以是改造翻修的已有的基础设施; 既可以运用在单 个的基础设施上, 也可以运用在基础设施群上。再次, 实地 安装的终端智能组件主要包括感测器和控制器两大类 : 感测 器用于探测当地实时的重要数据, 如温度、湿度、水量、风力、 土壤和水体成分等; 控制器主要进行实时的开关和调节等操 作。另外, 控制中心可以设在当地或者远程, 功能是通过通 信和计算智能组件来汇总信息、深度分析、下达指令。信息 主要来自于系统内部各类终端感测器以及系统外部有关气象 和水文的实时预报；数据的分析和处理通过设定的系统算法 完成。控制中心通过向终端控制组件发出调节和控制命令, 来完成对雨洪基础设施的实时维护、远程操作和科学管理。

\section{2 智能灰色和绿色基础设施对比}

智能技术主要应用于灰色和绿色两类主要基础设施有机 结合。智能灰色基础设施, 是运用智能技术在传感、控制、 交互通信等方面的优势对传统的水库、管道系统和处理厂进 行更新和改造, 以提高该系统的管理效率和能力。智能绿色 基础设施是一个主动的技术平台, 把感测器、控制器、通信 网络等智能组件结合到绿色基础设施的土壤、植被、工程结 构层, 完成实时操作和自动维护 ${ }^{[9]}$ 。

由于基础设施自身的不同特点和功能, 智能灰色和绿 色基础设施在规模、管理和形式上都有着显著差异（表 1)。 首先, 前者的规模相对较大, 多为集中系统; 后者规模相对

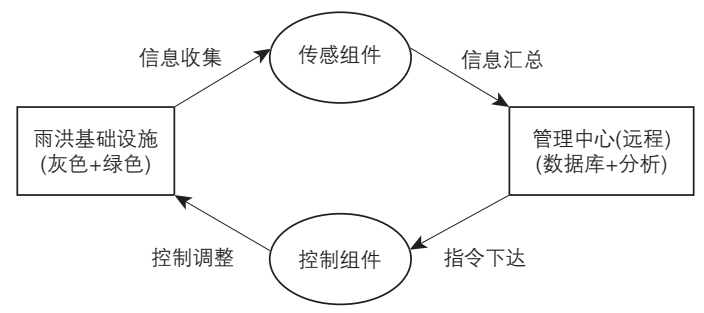

图 2 智能雨洪管理过程示意

资料来源 : 作者绘制
较小, 常见分散的小系统。传统的灰色基础设施多用于集中 控制和处理一个地区的雨洪量, 而绿色基础设施突出其对局 部雨洪的控制和管理。其次, 前者的管理重点在于提高系统 的处理能力, 重在对水量的控制; 而后者更多地关注对水质 的监测和提高。再次, 前者的形式相对统一, 而后者的智能 技术结合形式多样。智能技术在灰色基础设施上的应用主要 包括对管道的自动维护、水库的科学控制和水处理系统的实 时监控。由于绿色基础设施自身的多种类和当地化, 智能技 术与其结合形式也更为多样, 比如绿色屋顶的自动灌溉、可 透水路面的整体监控、人工湿地的水质分析等。

运用智能雨洪基础设施完成智能雨洪管理, 除了考虑 到两类基础设施的区别, 还应当注意以下几点。首先, 灰 色和绿色基础设施是两类不同的雨洪管理体系, 应该两者 结合运用: 在通过灰色基础设施对传统的雨洪管理系统实 施维护和升级的同时, 积极建造和推广适合当地的绿色基 础设施。这样, 一方面可以减少传统雨洪系统的压力, 另 一方面很好地解决了水域环境污染的问题。其次, 智能雨 洪管理是一种通过基础设施和智能技术的有效结合, 从而 达到主动、动态、实时、系统的管理和维护基础设施。通 过智能技术对基础设施的升级, 现有的传统技术设施的各 个子系统可以更好地结合, 绿色基础设施的功能和效率可 以有效地提高, 灰色和绿色基础设施也可以通过智能平台 很好地互相配合。

\section{3 智能雨洪管理的应用}

智能雨洪管理存在很大的发展和推广空间。这一部分结 合目前的应用实例从智能技术的应用现状出发, 分析智能雨 洪管理的发展方向和推广中的现实阻碍, 提出未来发展的有 效途径。

\section{1 应用现状}

目前的智能雨洪管理多集中于政策和监管的需要和学术 分析, 大规模采用和推广还相对有限。然而, 不乏一些有益 的探索和尝试。

首先, 在水量控制方面的探索。智能感测组件已经开始 运用到综合排水系统中, 用来监控、控制和减少不必要的未

表 1 智能灰色和绿色基础设施对比

\begin{tabular}{lll}
\hline & \multicolumn{1}{c}{ 智能灰色基础设施 } & \multicolumn{1}{c}{ 智能绿色基础设施 } \\
\hline 规模 & 较大, 集中 & 较小, 零散 \\
管理 & 水量的控制 & 水质的提高 \\
形式 & 相对统一 & 多样化 \\
监测对象 & 水库、管道 & 植被、土壤、工程设施等 \\
\hline
\end{tabular}

资料来源 : 作者绘制 
处理的雨污合流排放频率 ${ }^{[5]}$ 。在华盛顿州西雅图的一个试点 项目中, 一种智能算法综合考虑了系统的动态复杂性、不确 定的雨洪合流量、水库的适时监控, 达到了对雨污合流控制 的近似最优化管理 ${ }^{[10]}$ 。控制过量径流和蓄水节水的目标可以 由同一个智能技术的应用达到 ${ }^{[11]}$ 。安装在马里兰州最大的 炼煤厂附近的雨洪排放智能控制系统不仅可以有效降低雨 洪排放的频率和水量, 而且还将蓄水池的水运用于控制空 气煤尘 (图 3a $)^{[12]}$ 。

其次, 在水质监测方面的探索。集成无线感测器已经用 于水体质量的监控、控制和管理 ${ }^{[6]}$ 。智能感测计算用于监测 接收水域的污水径流排放情况和水质检测 ${ }^{[13]}$ 。模拟研究显示 将智能实时控制运用在保持水分上, 系统去除水中小颗粒的 能力较传统的设计提高了 $60 \%{ }^{[14]}$ 。在佛罗里达州中部进行的 有关人工雨洪池塘和湿地的研究中, 智能感测网络可以评测 控制侵蚀程度的能力, 监测地下污染物的迁移, 记录水利停 留时间等 ${ }^{[15]}$ 。

再次, 在提高系统功能和效率方面的探索。通过实时 监控、有预见性的控制、危机应对和反应, 以及自我维护 等相关智能技术的一系列用途, 智能技术在雨洪管理系统 中的应用有利于提高该系统的工作表现和弹性 ${ }^{[4]}$ 。在马里 兰大学的绿色屋顶实验项目中, 智能感测器网络被用来实 时监测绿色屋顶运行的各项参数, 包括水量蒸发率和径流 量控制的效率等, 有利于提高对绿色基础设施性能的全面 评估 ${ }^{[16]}$ 。在华盛顿环境保护局总部和科罗拉多州丹佛安装 的咜税器可以根据未来天气情况提前对水箱进行控制和管 理（图 3b,3c） ${ }^{[12]}$ 。

最后, 在环境和生态系统保护方面的探索。在绿色基础 设施和周边环境的实地研究中, 智能感测器用于实时和连续 地监测一系列的环境参数, 包括空气和土壤温度、相对湿度, 风速, 光合和太阳辐射, 降水量的数据 ${ }^{[16,17]}$ 。纽约布鲁克林 智能池塘的设计和运行充分考虑了降低环境影响和维护水域 生态系统的功能 (图 3d) ${ }^{[12 !}$ 。水利系统的最优化控制可以提 高能源效率、降低成本, 有利于环境保护 ${ }^{[18]}$ 。
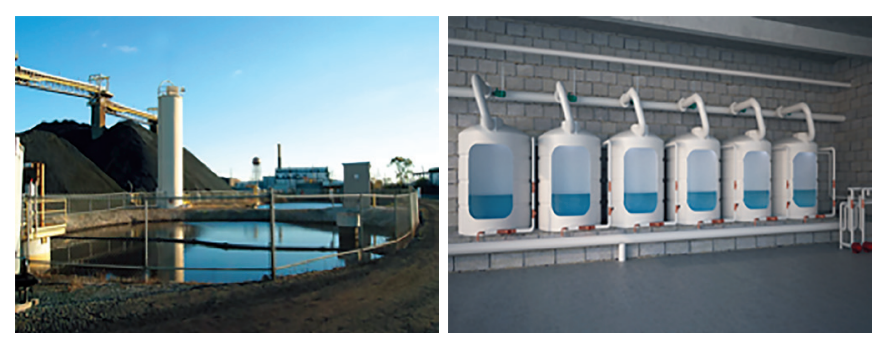

(a) 马里兰控制径流系统

(b) 华盛顿环保署智能水箱

图 3 部分 Opti 公司的智能雨洪基础设施试点项目 资料来源 : 参考文献 [12]

\section{2 发展重点}

基于智能雨洪管理的现有探索, 未来智能雨洪管理可以 在以下两个方面重点发挥作用。

（1）集成雨洪管理的资源和废物管理双重性。雨洪管理 本身具有很强的两面性, 是废物管理和资源管理的结合体。 当风暴来袭, 水量上涨, 过量的积水成为威胁人们日常生活 的隐患时, 雨洪管理重在废物废水管理, 需要及时快速清除 建筑环境中的积水, 维护人员财产的安全; 而面对持续干旱, 宝贵的雨水存续量成为维持人们生活的一道重要屏障时, 雨 洪管理重在资源管理, 需要将降水科学储存并合理应用于生 产生活的各个方面。智能雨洪管理的动态、主动、系统管理 的特性, 可以帮助现有的系统把水资源搜集和利用以及废水 废物处理有效结合起来, 完成双重角色的灵活转变。

（2）水量和水质的协同控制。雨洪管理不仅要管理和协 调水量, 还要监测和维护水质, 保护水域环境。极端天气状 况和快速城市化带来了大量的地表径流, 同时也造成了水质 的下降和水域环境的破坏。智能雨洪管理可以通过实时监测 参数、自动操作和维护系统, 预见性决策和控制, 极大发挥 现有雨洪基础设施的功能性, 更好地满足雨洪管理中对水量 和水质的要求。

\section{3 现实阻碍和推广途径}

尽管美国用于雨洪管理的智能技术本身已相对成熟，但 是目前的应用多集中于学术探讨、政策和监管的需要, 以及 有限的商业尝试。在学校和研究机构方面, 一些以水利和环 境工程专业闻名的大学 (比如宾夕法尼亚州的威兰偌瓦大学 和密西根大学) 正在积极进行有关智能雨洪管理的基础数据 收集、创新课题研究和校园试点项目建设等。在商业机构方 面, 为数不多的几家智能雨洪或者基础设施公司（比如 Opti 和 Bentley）积极参与和实施雨洪基础设施的智能升级, 包括 项目设计、实地安装、后期维护和评估等业务, 已经在全国 范围内开展了许多试点项目。在政府部门和管理机构方面, 联邦和地方两级政府还没有出台明确的有关智能雨洪管理的
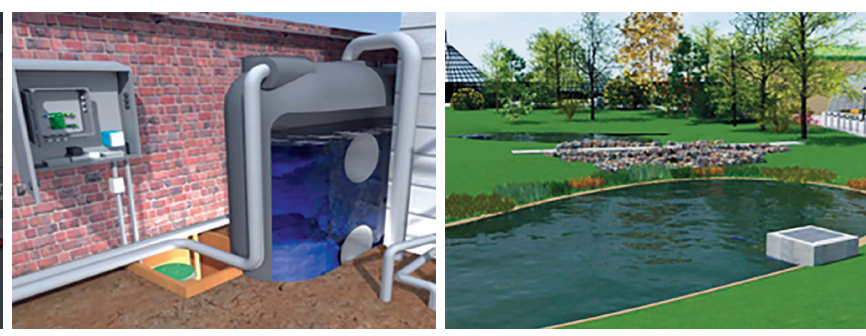

(c) 科罗拉多绿色学校贮水器 
政策和管理文件, 目前的引导措施多在于对研究项目的资金 支持和对试点项目建设的鼓励。

阻碍智能雨洪管理大规模实地采用和推广的因素有以下 几点。首先, 受限于雨洪管理系统本身的复杂性。水利基础 设施和设备的种类庞杂, 各地降水和雨洪管理的具体情况多 样, 实际的管理机构和主体各异, 都给智能技术和雨洪管理 的有效结合和进一步推广造成了阻碍。其次, 缺少明确有力 的政策支持。虽然联邦政府出台的相关法规明确了雨洪的管 理职责和要求，但是地方政府对雨洪系统未来的规划发展方 向和指导还不明确。由于雨洪基础设施自身的公共产品特性 和进行系统研发需要投人的大量资金, 都制约着智能雨洪管 理的进一步开发、推广、应用。再次, 相关市场和商业通道 还没有完全建立。目前市场上只有零星几家公司在做与水利 相关的商业智能组件和产品的探索, 从产品研发到推向市场, 再到用户反馈的商业通道还远没有建立起来。

未来美国智能雨洪管理推广的重点在于完善智能管理的 框架和思路, 分析各地各部门对雨洪管理的具体需要, 研发 多样的智能基础设施系统, 完成技术和基础设施的有机结合, 探索有效的发展和推广途径。

第一，市场的建立和运营。成熟和健康的市场环境是智 能雨洪管理发展的重要平台。首先，技术的设计和研发要充 分听取用户的需求。智能技术广泛采用和推广极大程度取决 于其能否很好满足工作在雨洪管理第一线的机构和人员的需 要 ${ }^{181}$ 。其次, 合理的技术组合和产品定价。产品定价、功能 组合、长期维护和修理成本都对技术的成功推广起着至关重 要的作用。再次, 相关市场的建立和成熟。需要鼓励涉及智 能系统维护和操作的新型公司的成立, 并有计划地建立雨洪 管理的智能组件的相关市场。

第二, 政策引导和支持。基于雨洪管理的公共产品属性, 政策和市场需要互相配合, 共同发挥作用。对于智能雨洪管 理这一新兴的理念和系统, 政府应当积极参与到科研和实际 推广中, 制定合理的发展思路和框架, 将智能雨洪和城市的 可持续发展紧密联系。具体包括 : 在智能雨洪产品和系统进 人市场的初期, 可以对相关的企业和产品提供政策扶持 ; 在 市场失灵的水环境治理和生态系统恢复等相关领域，制定合 理的政策标准, 动用公共资源, 建立示范点, 完善奖罚机制。

\section{4 对我国雨洪管理智能化的借鉴意义}

我国目前的雨洪管理同样面临着严峻的考验。全球气候 变化直接影响了降水和水资源分布, 快速城镇化带来了严重 的水污染和水控制问题, 环境问题的突显和生态系统维护的 意识加强对雨洪管理提出了新要求 ${ }^{[20.22]}$ 。通过科学的雨洪管 理应对极端天气和自然灾害，控制城市径流，降低水污染和
保护水环境显得尤为重要。然而, 我国目前传统的雨洪控制 利用系统, 存在着资源利用理念落后、控制利用模式单一、 决策和管理相对滞后等突出问题 ${ }^{23]}$ 。智能雨洪管理在美国的 尝试、发展和经验, 都将对中国的雨洪管理智能化的潜在发 展提供重要的借鉴意义。

第一, 智能技术的开发利用要和各地区雨洪管理的实际 需要紧密联系。美国东北部的城市多存在雨污合流带来的水 体污染问题, 故智能雨洪管理的重点放在如何准确检测水质 参数和降低水体沉积物; 而沿海地区的智能雨洪需求更偏重 对极端天气的预警和防范。智能雨洪管理未来在我国的发展 和应用要甄别和明确各地的具体需求。雨洪管理的重点是控 制水量, 还是监测水质, 是快速排洪, 还是有效蓄水, 都要 充分结合当地的实际需要。

第二, 充分发挥智能雨洪管理在实时操作和系统管理中 的优势, 有效升级已有的水利基础设施。对雨洪管理的智能 优化可以在不新建或者扩建已有雨洪管理系统的基础上, 通 过安装智能组件和远程分析计算, 对零散的基础设施实施网 络化系统管理, 有效地提高现有系统的功能性。这样可以降 低系统更新升级的成本, 合理利用现有的设备和资源, 极大 提高雨洪管理的效率。

第三, 智能雨洪管理系统应当做到灵活运用和自动演进。 雨洪系统的智能化不要局限于已有的智能技术和应用模式, 而是应当充分参与智能技术的研发和应用探讨, 不断结合新 的需要改善和创新智能雨洪系统。一方面, 汇总的来自雨洪 管理系统的信息和数据的种类和频率需要随着管理需要和外 部环境自动增减和变化。比如, 水域附近发生突发的重金属 污染, 水质的监测和控制参数应当及时纳人该种重金属和相 关的水体成分的变化。随着极端天气的强调变化, 智能数据 收集和分析频率也应当随之变化。通过提高雨洪管理系统的 韧性, 结合系统内外的信息有效沟通和处理, 对极端天气、 自然灾害, 以及突发事件提前进行预防性控制。

未来，我国可以借鉴美国以及其他国家地区的雨洪管理 理念和技术, 结合自身的国情和需要, 完善我国的雨洪管理 体系。 UP

\section{参考文献}

[1] GRIFFIN D, ANCHUKAITIS K J. How unusual is the 2012-2014 California drought?[J]. Geophysical Research Letters, 2014, 41(24): 9017. 9023.

[2] LEE J G. Estimation of urban imperviousness and its impacts on storm water systems[J]. Journal of Water Resources Planning and Management, 2003, 129(5): 419-426.

[3] THURSTON H. Opportunity costs of residential best management practices for stormwater runoff control [J]. Journal of Water Resources Planning and Management, 2006, 132(2): 89-96. DOI: 10.1061/(ASCE)0733. 9496(2006)132:2(89). 
[4] RASEHK A, HASSANZADEH A, MULCHANDANI S, et al. Smart water networks and cyber security[J]. Journal of Water Resources Planning and Management, 2016,142(7): 01816004-1. DOI: 10.1061/(ASCE)WR.19435452.0000646 .

[5] RUGgaber T P, TAlley J W, Montestruque L A. Using embedded sensor networks to monitor, control, and reduce CSO events: a pilot study [J]. Environmental Engineering Science, 2007, 24(2): 172-182.

[6] ZIA H, HARRIS N R, MERRETT G V. Water quality monitoring, control and management framework using collaborative wireless sensor networks[C/ $\mathrm{OL}]$. Conference presented at the 11th International Conference on Hydroinformatics (HIC), 2014. http://eprints.soton.ac.uk/365852/.

[7] KERKEZ B, GRUDEN C, LEWIS M, et al. Smarter stormwater systems [J]. Environmental Science \& Technology, 2016, 50: 7267-7273.

[8] STEINER F, SIMMONS M, GALLAGHER M, et al. The ecological imperative for environmental design and planning[J]. Frontiers in Ecology and the Environment, 2013, 11(7): 355-361.

[9] MENG T, HSU D, WADZUK B. Green and smart: perspectives of city and water agency officials in Pennsylvania toward adopting new infrastructure[J]. Journal of Sustainable Water in the Built Environment, 2017, 3(2): 05017001.

[10] DARSONO S, LABADIE J W. Neural-optimal control algorithm for realtime regulation of in-line storage in combined sewer systems[J]. Environmental Modelling \& Software, 2007, 22(9): 1349-1361.

[11] REIDY P C. Innovation in CSO reduction: implementing intelligent distributed infrastructure[C]. World Environmental and Water Resources Congress 2011: Bearing Knowledge for Sustainability, 2011: 3539-3549.

[12] Opti. Project[EB/OL]. (2017)[2017-8-18]. http://optirtc.com/projects.

[13] YU B, BEHERA P K, ROCHAC J F R. Advanced sensor-computer technology for urban runoff monitoring[J]. Proceedings of SPIE, 2011, 79815U. DOI: $10.1117 / 12.881817$

[14] MUSCHALLA D, VALLET B, ANCTIL F, et al. Ecohydraulic-driven realtime control of stormwater basins[J]. Journal of Hydrology, 2014, 511(4): 82-91.

[15] CRAWFORD A. Green technologies and sensor networks for BMP evaluation in stormwater retention ponds and wetlands[D/OL]. (2014) [2017-8-7]. Master thesis. University of Central Florida. http://stars.library. ucf.edu/etd/1209/.

[16] STARRY O, LEA-COX J, RISTVEY A, et al. Monitoring and modeling green roof performance using sensor networks[]]. Acta Horticulturae, 2014, 1037(1037): 663-669.

[17] STARRY O S, LEA-COX J D, RISTVEY A G, et al. Utilizing sensor networks to assess evapotranspiration by greenroofs[C]. American Society of Agricultural and Biological Engineers, 2011.

[18] OCAMPO-MARTINEZ C, PUIG V, CEMBRANO G, et al. Application of predictive control strategies to the management of complex networks in the urban water cycle[J]. IEEE Control Systems, 2013, 33(1): 15-41. DOI:10.1109/MCS.2012.2225919.

[19] WANG Z, SONG H, WATKINS D W, et al. Cyber-physical systems for water sustainability: challenges and opportunities[J]. IEEE Communications Magazine, 2015, 53(5): 216-222. DOI:10.1109/MCOM.2015.7105668.

[20]王岩. 论海绵城市在市政工程设计中的应用 [J]. 城市道桥与防洪, 2016, 1: 100-102.

[21] 张静. 中国科学院院士刘昌明: 海绵城市建设需做足气候变化应对文 章 [N]. 中国气象报, 2016-2-17(1).

[22] 潘安君, 张书函, 孟庆义, 等. 北京城市雨洪管理初步构想 [J]. 中国给 水排水, 2009, 25(22): 9-12.

[23] 车伍, 张伟, 李俊奇, 等. 中国城市雨洪控制利用模式研究 [J]. 中国给 水排水, 2010, 26(16): 51-57.

(本文编辑：许玫) 